\title{
Láser en odontología: fundamentos físicos y biológicos Laser in Dentistry: Physical and Biological Foundations
}

Jhon Fredy Briceño Castellanos ${ }^{1}$

Diego Alejandro Gaviria Beitia ${ }^{\text {a }}$

Instituto de Tecnología Avanzada, México

gaviriadoc@hotmail.com

Yurani Angélica Carranza Rodríguez ${ }^{2}$

DOI: https://doi.org/10.11144/Javeriana.uo35-75.loff Redalyc: http://www.redalyc.org/articulo.oa? id $=231249121007$

Fecha de recepción: 30 Junio 2016 Fecha de aprobación: 23 Diciembre 2016

\section{Resumen:}

Antecedentes: El láser es una tecnología cada vez más utilizada en odontología. Para tomar decisiones acertadas con respecto a las características y uso del láser es importante conocer sus bases físicas y biológicas, en cuanto a su interacción con los tejidos. Objetivo: Analizar los fundamentos biológicos y físicos del láser en odontología. Métodos: Se llevó a cabo una revisión narrativa con base en literatura publicada entre 1990 y 2016 e incluida en el Medline. La muestra consistió en 30 artículos. Para el análisis se empleó un enfoque hermenéutico. Resultados: Los fundamentos físicos analizados incluyen luz, amplificación, emisión estimulada y radiación. En cuanto a los efectos biológicos, se analizan el fototérmico, el fotoquímico y el fotoacústico. Asimismo, se describen las propiedades ópticas de los tejidos orales: absorción, penetración y longitud de extinción. Conclusiones: No todos los láseres actúan igual y una misma longitud de onda puede tener interacciones diferenciales en los tejidos. Existen varios estudios que evidencian la efectividad del láser en varias especialidades de la odontología y abren la posibilidad de varias líneas de investigación.

Palabras clave: absorción, amplificación, efecto fotoacústico, efecto fotoquímico, efecto fototérmico, emisión estimulada, láser, longitud de extinción, luz, odontología, penetración del rayo, radiación, tecnología.

Áreas Temáticas: biofísica; odontología; respuesta biológica; biotecnología.

\section{Abstract:}

Background: Laser technology usage is increasing in dentistry. In order to take adequate decisions about characteristics and use, it is important to know the physical and biological foundations of laser and its interaction with oral tissues. Objective: To analyze the physical and biological foundations of laser in dentistry. Methods: A narrative review of literature published between 1990 and 2016 and included in Medline was carried out. The sample consisted of 30 articles. Analysis of literature was performed through a hermeneutical approach. Results: Physical foundations of laser analyzed include light, amplification, stimulated emission, and radiation. Biological effects studied are photothermal, photochemical, and photoacoustic. In addition, the article describes optical properties of oral tissues such as: absorption, penetration, and extinction length. Conclusion: Not all lasers act the same way and the same wave length can interact differently with each tissue. Several studies show evidence of the effectiveness of laser in several dental specialties and open the possibility for several lines of research.

Keywords: absorption, amplification, dentistry, extinction length, laser, light, photoacoustic effect, photochemical effect, photothermal effect, radiation, ray penetration, stimulated emission, technology.

Thematic fields: biological response; biophysics; biotechnology; dentistry.

\section{INTRODUCCIÓN}

En 1960, Theodore Maiman, un científico de la corporación Hughes Aircraft, desarrolló el primer dispositivo láser que emitía un haz de luz rojo profundo desde un cristal de rubí. Desde ese entonces, el dispositivo láser ha evolucionado y sus usos se han expandido. Una de las áreas donde se ha extendido son las ciencias biomédicas,

Notas de autor:

\footnotetext{
a Autor de correspondencia. Correo electrónico: gaviriadoc@hotmail.com 
particularmente la odontología [1,2,3,4]. Leon Goldman, un dermatólogo que había experimentado con la remoción de tatuajes, fue el primer científico que utilizó el láser de rubí sobre el diente de su hermano odontólogo, en 1965. El resultado fue un esmalte con dolor y fracturado $[5,6]$.

A partir de ese momento, se desarrollaron en los años setenta y ochenta diferentes tipos de láser y se llevaron a cabo estudios sobre su interacción con diferentes tejidos. La mayoría de las investigaciones usaron el láser de dióxido de carbono y neodimio: granate de aluminio de itrio (Nd:YAG) para remover tejidos blandos y en procedimientos periodontales (en 1987, se le otorgó permiso a Myers y Myers para utilizar el Nd:YAG en procedimientos periodontales). Desde ese entonces, numerosos dispositivos láser se encuentran disponibles para la práctica dental, además de varios que se encuentran en desarrollo [1,2,3,7]. El uso del láser ha permeado las principales ramas de la odontología y se encuentran diversos estudios que abordan diferentes aplicaciones de la terapéutica fotónica en esta área. Comparar sus resultados y aplicaciones con las terapias convencionales es controversial $[4,5]$.

Por otro lado, se ha avanzado en el entendimiento del funcionamiento del láser y de las propiedades ópticas de los tejidos en las diferentes disciplinas biomédicas, aunque todavía falta claridad sobre las bases físicas del láser y su interacción con los tejidos [7]. Por ello el objetivo de este artículo fue analizar los fundamentos físicos y biológicos de la tecnología láser en odontología.

\section{MATERIALES Y MÉTODOS}

Este estudio fue una revisión narrativa de la literatura que se realizó en dos fases: una heurística y una hermenéutica. En la primera fase se llevó a cabo una búsqueda inicial de artículos publicados entre enero de 1990 y mayo del 2016 que estuvieran incluidos en Medline. La estrategia de búsqueda se enfocó en las bases biológicas y físicas de la odontología láser. Así, se incluyeron los siguientes términos, solos o en combinación, estandarizados en el tesauro Medical Subject Headings ( $\mathrm{MeSH}$ ): ("lasers" [MeSH Terms] OR "lasers" [All Fields] OR "laser" [All Fields]) AND ("dentistry" [MeSH Terms] OR "dentistry" [All Fields]) AND ("foundations" [MeSH Terms] OR "foundations" [All Fields] OR "foundation" [All Fields]). Como criterios de inclusión se tomaron artículos presentes en revistas científicas arbitradas de odontología, escritos en inglés y español, que incluyeran en su contenido el láser como opción quirúrgica y terapéutica. Se excluyeron aquellos artículos que no hablaran de la fundamentación biológica ni física del láser.

Después se realizó una fase hermenéutica, donde se efectuó una lectura exhaustiva y crítica de la documentación disponible, para crear una recopilación de la información disponible y así agrupar los contenidos temáticamente. La fase hermenéutica se dividió en recopilación de la información, almacenamiento en fichas o unidades de información, categorización de unidades informáticas y temáticas, elaboración del esquema conceptual o listado esquemático de contenidos de la producción escrita, enlace y coordinación de unidades informáticas y temáticas que permitió mantener la ilación y coherencia de los diferentes contenidos y redacción del texto final del trabajo [8].

Como se describió, se buscó, recopiló y organizó un inventario de artículos, para conformar una base de datos y construir así una bibliografía temática y metodológica inicial. De la fase heurística se obtuvieron 114 títulos. Durante la fase hermenéutica se revisaron todas las referencias encontradas que se agruparon en dos grandes temas: bases físicas del láser y bases biológicas de la interacción láser-tejidos. Así, la muestra final incluyó un total de 30 artículos.

Cabe aclarar que este trabajo no debe ser considerado una revisión sistemática, sino una revisión cualitativa, hecha bajo los parámetros de un estado del arte, ya que no puede asumirse como un producto terminado, sino como una contribución que genera nuevos problemas o nuevas hipótesis de investigación y representa un primer insumo para comenzar una investigación [6]. Por ello, este artículo representa una actividad investigativa inicial para explorar lo que se ha dicho, cómo se ha dicho, a fin de ser el primer esfuerzo en realizar 
una descripción, explicación o interpretación del láser en odontología. Así, se estructura el documento en dos apartados generales: los fundamentos físicos donde se nombran las características de los láseres utilizados en odontología y los fundamentos biológicos que incluyen las interacciones de los láseres con los tejidos.

\section{RESULTADOS}

\section{Fundamentos físicos del láser}

La palabra láser es el acrónimo de "amplificación de luz por emisión estimulada de radiación” (del inglés light amplification by stimulated emission of radiation). Es un proceso por el cual la energía eléctrica es convertida en energía lumínica, que se origina por la excitación de átomos de un material láser y dispara una emisión espontánea de fotones $[2,5,6,9]$. A continuación, se realiza un recuento breve de los términos principales que permitirán explicar los principios físicos del láser.

\section{$L u z$}

La luz es una forma de energía electromagnética que viaja a una velocidad constante y se puede comportar como una onda o una partícula. La unidad fundamental de la luz se denomina fotón. Esta se puede definir a partir de dos propiedades: la amplitud y la longitud de onda. La amplitud se define como el tamaño total de la oscilación de onda desde la punta superior del pico hasta la parte inferior en un eje vertical e indica la cantidad de intensidad de la onda. Así, entre más amplitud, más cantidad de trabajo útil que se puede realizar $[2,6,10]$ (figura 1 ).

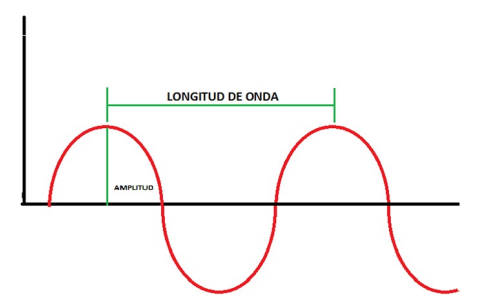

FIGURA 1

Propiedades de la onda electromagnética

Por otra parte, la longitud de onda se define como la distancia entre dos puntos correspondientes de la onda en el eje horizontal. La longitud de onda indica cómo la luz láser se libera al sitio quirúrgico y cómo reacciona con el tejido. La longitud de onda se expresa en micrones o nanómetros. Una propiedad de la longitud de onda es la frecuencia (número de oscilaciones de onda por segundo y es inversamente proporcional a la longitud de onda). El hertzio ( $\mathrm{Hz})$ se define como el número de pulsos de láser emitidos por segundo; mientras un pulso se define como la emisión de luz en forma de destellos $[11,12,13]$.

La luz láser presenta algunas características que la diferencian de la luz ordinaria. Este tipo de luz, como la emitida por linternas, presenta un resplandor blanco difuso que consiste en la suma de muchos colores del espectro visible (violeta, azul, verde, amarillo, naranja y rojo). Se puede utilizar un prisma para separar los colores individualmente, de la misma manera que las gotas de lluvia rompen la luz solar en los colores del arcoíris. Por su parte, la luz láser refiere un color específico, es monocromática, puede ser visible o invisible, de espectro continuo y con poca longitud de onda $[1,2,3,4,5,6,7,8,9,10,11,12,13,14,15]$.

Las propiedades específicas que caracterizan la luz láser son: colimación (haz con límites espaciales específicos, la cual asegura un tamaño y una forma de onda constante emitida de la cavidad láser), coherencia (las ondas de luz producidas en un instrumento son las mismas, sincronizadas y en las mismas formas, es decir, picos y valles equivalentes) y eficiencia (a menor potencia, mayor aprovechamiento de energía para el efecto deseado) [16]. 


\section{Amplificación}

La amplificación delimita el proceso que sucede en el interior del láser y determina cómo se produce la luz láser. El centro del láser se denomina cavidad láser y los componentes que la producen son el medio activo, el mecanismo de bombeo y el resonador óptico. El medio activo se define como los elementos químicos que pueden ser cristales sólidos, gases, líquidos o semiconductores que, al aplicarles energía atómicamente, se estimulan en sus capas internas y se tornan inestables. Inmediatamente, al buscar su estabilización, se libera energía en forma de fotones $[1,2,5,10]$ (figura 2).

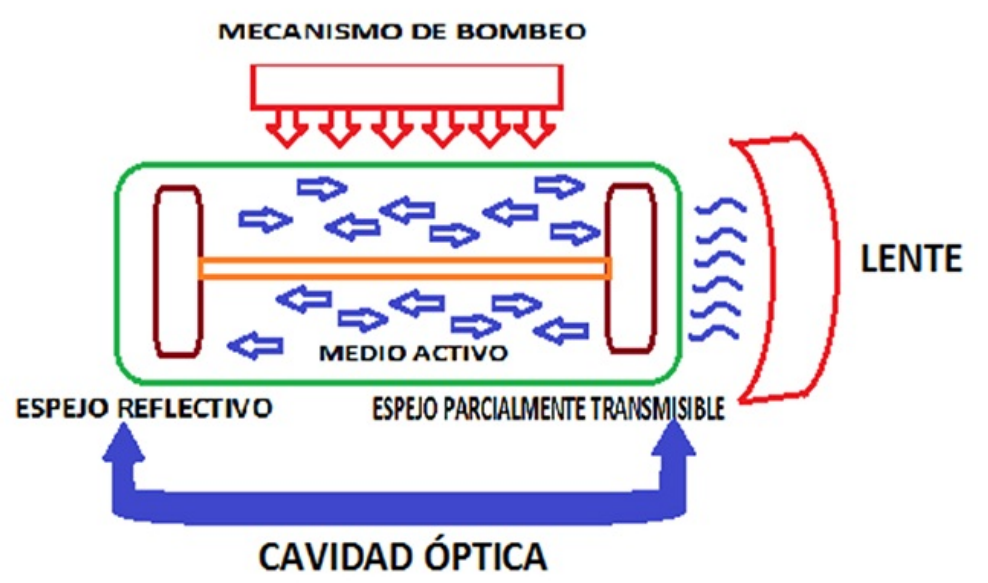

\section{FIGURA 2}

Elementos de la cavidad óptica que permiten la amplificación de la luz láser

El mecanismo de bombeo es aquella fuente de energía externa (que pueden ser sistemas eléctricos, lámparas de luz o bobinas eléctricas) que transmite energía a los átomos para excitarlos en la capa más externa de los átomos del medio activo. Estos electrones externos admiten una cantidad de energía y pasan al nivel más lejano del núcleo, que están a un nivel de energía más alto. Allí se produce un fenómeno denominado inversión de población; en estos átomos se observan más electrones con energía alta que electrones con energía inicial [17]. Posteriormente, los electrones emiten la energía espontáneamente en forma de fotón en un proceso denominado emisión espontánea. La cavidad láser presenta dos espejos, uno a cada extremo de la cavidad láser, en disposición paralela, que actúan como resonadores ópticos, y cuya función es reflejar las ondas hacia adelante y atrás y ayudar a colimar/amplificar el nuevo haz de luz $[1,2,5,10]$.

\section{Emisión estimulada}

La emisión estimulada se define como el proceso por el cual los haces de luz láser se producen dentro de la cavidad láser. Este fenómeno se describe a partir de la teoría de cuerpo negro de Albert Einstein, de 1916, que describe la transcripción espontánea de fotones desde las teorías de Max Planck y Niels Bohr. Según esta, la energía se irradia como fotones en una onda coherente; así la emisión hace que el haz de luz se expanda/ amplifique geométricamente y da la posibilidad de cuantificar la energía $[6,7,14]$. Einstein propuso que en este intercambio de electrones entre el exterior y el átomo pueden existir dos procesos: absorción o emisión. A su vez, dentro de los procesos de emisión existen dos fenómenos: emisión espontánea (de un fotón a partir de la desestimulación espontánea del átomo) y emisión estimulada (de dos fotones similares a partir de un fotón incidente). Esta última clase de emisión es el principio físico del láser [2,4,10] (figura 3). 


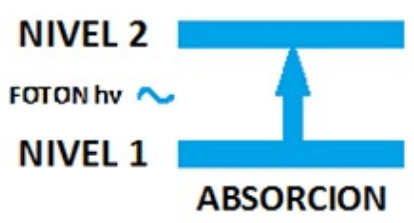

ABSORCION

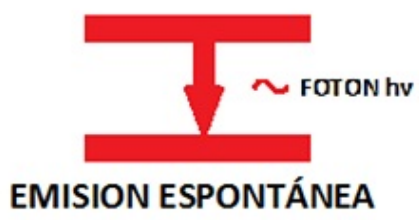

FIGURA 3

Procesos de absorción-emisión descritos por Einstein en 1916

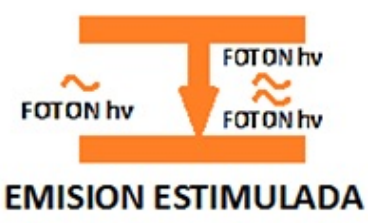

EMISION ESTIMULADA

\section{Radiación}

Las ondas de luz son una forma específica de radiación o energía electromagnética. El espectro electromagnético en odontología maneja ondas no ionizantes, con longitudes de onda que oscilan entre 0,5 $\mu \mathrm{m}(500 \mathrm{~nm})$ y $10,6 \mu \mathrm{m}(10600 \mathrm{~nm})$. Estas longitudes de onda están ubicadas en la luz infrarroja en una porción denominada radiación térmica $(0,7-10,6 \mu \mathrm{m})$. Por otra parte, las longitudes de onda ubicadas en el rango ultravioleta (menor de $500 \mathrm{~nm}$ ) presentan un efecto ionizante. En otras palabras, producen secuelas mutagénicas en el ADN celular, razón por la cual poco se utilizan en odontología directamente sobre tejidos con fines terapéuticos $[1,4,10,14,15,18]$.

\section{Fundamentos biológicos del láser}

Adicionalmente a los fundamentos físicos del funcionamiento del láser, se debe tener en cuenta la respuesta de los tejidos a la luz láser. Los láseres en odontología se pueden dividir dependiendo de la longitud de onda a la cual pertenecen: láseres rojos y láseres infrarrojos. Al grupo de láseres rojos o visibles $(350-750 \mathrm{~nm})$ pertenecen los de argón (488-514 nm) y potasio titanil fosfato (KTP de $532 \mathrm{~nm}$ ). Estos láseres se ven en el campo odontológico en diversas aplicaciones como $635 \mathrm{~nm}$ para detección de caries (Diagnodent ${ }^{\circ}$ ) y algunos láseres de diodo utilizados para terapia de baja intensidad (LLLT) como el $660 \mathrm{~nm}$ (Argilaser ${ }^{\circ}$ ) $[1,2,5]$.

Existen otros láseres dentales que pertenecen a la porción cercana, media o lejana del espectro infrarrojo. Estos tipos de láser no son visibles al ojo humano, por lo que se requieren haces de luces visibles como apuntadores. Estos tipos de láser del infrarrojo cercano incluyen diodos $(800-980 \mathrm{~nm})$ y neodimio: itrio aluminio y granate Nd:YAG (1064 nm), los cuales usan un medio activo semiconductor, son poco afines al agua y su mayor absorbancia se presenta a pigmentos presentes en sangre y tejidos denominados cromóforos, por ejemplo, hemoglobina y melanina. Adicionalmente, los láseres ubicados en la porción infrarroja media Er:YAG $(2940 \mathrm{~nm})$ o ErCr:YSGG $(2780 \mathrm{~nm})$ y láser del infrarrojo lejano $\mathrm{CO}_{2}(10.600 \mathrm{~nm})$ son más afines al agua y poco afines a cromóforos $[1,2,5]$.

Para poder avanzar en el entendimiento de la terapéutica láser en los tejidos orales, se deben comprender, en primer lugar, los efectos cuando este interactúa el láser. Dependiendo de la temperatura que alcance el tejido, se deben distinguir dos grandes grupos de láser: los duros y los blandos. Los blandos o soft laser (LLLT por lowlevel laser therapy) no producen aumento de temperatura y generan efectos directos sobre la cicatrización y la regeneración celular. Se les denomina efectos bioestimuladores. La base de la terapia LLLT es que su actividad sobre los tejidos no obedece a efectos térmicos, sino a la interacción de las ondas electromagnéticas de esta radiación con las células. La energía se absorbe donde la concentración de fluidos es mayor. Por lo tanto, habrá una mayor absorción en los tejidos inflamados y edematosos, estimulando las numerosas reacciones biológicas relacionadas con el proceso de reparación de las heridas $[1,4,5,17]$ (tabla 1). 
TABLA 1

Principales tipos de láser utilizados en odontología

\begin{tabular}{|c|c|c|c|}
\hline Tipo de láser & $\begin{array}{l}\text { Longitud de } \\
\text { onda (nm) }\end{array}$ & Forma de onda & Aplicaciones \\
\hline $\begin{array}{l}\text { Dióxido de } \\
\text { carbono }\end{array}$ & 10600 & $\begin{array}{l}\text { Continuo } \\
\text { superpulsado }\end{array}$ & $\begin{array}{l}\text { Incisión y ablación de tejidos blandos } \\
\text { Desepitelialización gingival durante } \\
\text { procedimientos regenerativos } \\
\text { periodontales }\end{array}$ \\
\hline $\begin{array}{l}\text { Neodimio: } \\
\text { itrio- } \\
\text { aluminio- } \\
\text { granate } \\
\text { (Nd:YAG) }\end{array}$ & 1064 & Pulsado & $\begin{array}{l}\text { Incisión y ablación de tejidos blandos } \\
\text { Vaporización de caries incipientes } \\
\text { Hemostasia } \\
\text { Tratamiento hipersensibilidad dentinaria } \\
\text { Descontaminación periodontal } \\
\text { Descontaminación endodóntica }\end{array}$ \\
\hline $\begin{array}{l}\text { Erbio, itrio- } \\
\text { aluminio- } \\
\text { granate } \\
\text { (Er:YAG) }\end{array}$ & 2940 & Pulsado & $\begin{array}{l}\text { Incisión y ablación de tejidos blandos } \\
\text { Tratamiento hipersensibilidad dentinaria } \\
\text { Remoción de caries } \\
\text { Ablación de tejidos duros } \\
\text { Descontaminación periodontal } \\
\text { Descontaminación endodóntica }\end{array}$ \\
\hline $\begin{array}{l}\text { Erbio, cromo: } \\
\text { itrio-selenio- } \\
\text { galio-granate } \\
\text { (ErCr:YSGG) }\end{array}$ & 2780 & Pulsado & $\begin{array}{l}\text { Incisión y ablación de tejidos blandos } \\
\text { Tratamiento hipersensibilidad dentinaria } \\
\text { Remoción de caries } \\
\text { Ablación de tejidos duros } \\
\text { Descontaminación periodontal } \\
\text { Descontaminación endodóntica }\end{array}$ \\
\hline Argón & $457-502$ & Pulsado continuo & $\begin{array}{l}\text { Fotocurado de resinas } \\
\text { Activación de peróxido de carbamida } \\
\text { Incisión y ablación de tejidos blandos } \\
\text { Hemostasia }\end{array}$ \\
\hline
\end{tabular}

Los láseres duros, llamados quirúrgicos o de alta potencia (HLLT, por high level laser therapy), producen un efecto térmico sobre los tejidos, lo cual se traduce en cortes muy precisos, vaporización y coagulación de vasos de pequeño calibre $[1,4,5,17]$. La base de los efectos del láser quirúrgico sobre los tejidos es la conversión de la energía lumínica en energía térmica en su seno calentando y produciendo lesiones que dependerán de la temperatura alcanzada [9,19]. Este hecho depende tanto de las características del haz láser administrado como de las características del tejido sobre el que actúe. Para ello se deben tener considerar dos elementos: la longitud de onda y las propiedades ópticas de los tejidos $[1,2,4,5,7,10,11,20]$. Independientemente del tipo de láser, las interacciones del láser en los tejidos pueden darse mediante reflexión (redireccionamiento del láser sin efecto en los tejidos), transmisión (penetración de la luz sin efecto sobre el tejido), dispersión (la energía se disemina, debilitando su potencial de acción) y absorción (la energía es direccionada en su totalidad sobre el tejido) $[1,2,4,5,10]$.

El efecto terapéutico se logra cuando el haz de láser es absorbido por los tejidos, por lo que las interacciones fotobiológicas con los tejidos son las que realmente definen el resultado final. Según Smith [11], la primera ley de la fotoquímica es que la luz debe absorberse para que haya algún cambio en el tejido. La segunda ley habla de que no todas las longitudes de onda producen los mismos cambios fotobiológicos en los tejidos. Los principales efectos fotobiológicos pueden ser fototérmicos, fotoquímicos y fotoacústicos. Al respecto, pueden existir varias interacciones al mismo tiempo que dependen de los parámetros que se apliquen al láser [4]: 


\section{Efecto fototérmico}

La interacción fototérmica se caracteriza por un aumento de la temperatura local inducida por la acción del láser, característica distintiva de los láseres quirúrgicos [4]. Así, las principales interacciones fototérmicas son incisión/escisión de tejidos, ablación/vaporización y hemostasia/coagulación [1,2,4,5,10]. La energía de luz se contacta con el tejido por un tiempo determinado y produce una interacción térmica. Dependiendo de la temperatura alcanzada, el efecto varía. Cuando la temperatura se encuentra entre $37^{\circ} \mathrm{C}$ y $50{ }^{\circ} \mathrm{C}$, se produce inactivación bacteriana, muy útil en procesos periodontales y endodónticos. Allí es donde se han reportado varios estudios que apoyan la efectividad de la terapia láser como descontaminante de alto nivel en tejidos periodontales y endodónticos $[21,22,23,24,25]$.

Cuando la temperatura se encuentra entre $60{ }^{\circ} \mathrm{C}$ y $70{ }^{\circ} \mathrm{C}$, se observa coagulación y desnaturalización de proteínas. Así, cuando la temperatura aumenta a $100^{\circ} \mathrm{C}$, se produce vaporización de agua en un fenómeno que se denomina ablación. Varios estudios muestran mayor capacidad de cicatrización y mejores efectos postoperatorios cuando se comparan las terapias láser con otras terapias, ya que muestran simultáneamente efectos analgésicos, antinflamatorios, cicatrizantes y hemostáticos que no se observan con otras técnicas $[26,27,28,29,30,31]$. Cuando el proceso térmico sobrepasa los $200^{\circ} \mathrm{C}$, se produce un efecto denominado carbonización, donde el carbón se desarrolla como producto final y actúa como disipador del calor, causando trauma en los tejidos adyacentes $[1,2,5,10]$.

\section{Efecto fotoquimico}

Se estimulan reacciones químicas como el fotocurado de una resina, efectos de fluorescencia para detección de caries o la denominada terapia fotodinámica en la cual, gracias a la interacción de una sustancia sensibilizadora (generalmente un pigmento afín al láser) con el láser, se produce un radical de oxígeno con propiedades específicas, que ayuda a desinfectar bolsas periodontales y canales endodónticos $[1,2,5,10]$.

\section{Efecto fotoacústico}

Este efecto produce una onda de choque con acción vibratoria. Algunos estudios han reportado su efecto en odontología operatoria para el retiro de caries [1,2,4] y la descontaminación endodóntica [31] mediante vibración para remoción de la capa de barrillo, caries y bacterias sin contacto directo con el tejido.

\section{Propiedades ópticas de los tejidos orales}

Al tener en cuenta las propiedades ópticas de los tejidos, es preciso considerar dos características: la absorción y la penetración [18].

\section{Absorción}

Los principales elementos orgánicos que absorben energía en los tejidos dependen de la longitud de onda del láser que se maneje. Para los láseres ubicados en el espectro del cercano infrarrojo, la afinidad se encuentra en elementos pigmentados como la hemoglobina y la melanina, las cuales se denominan cromóforos. Por otra parte, los láseres ubicados en el espectro infrarrojo presentan mayor afinidad al agua de los tejidos [1,2,4,5,18]. 


\section{Penetración}

La boca es un órgano que presenta gran cantidad de tejidos con diversas propiedades ópticas, pero adicionalmente la interacción entre estos puede presentar respuestas diferenciales a diversos estímulos. Por ello, además de la absorción por parte de los tejidos, se debe tener en cuenta que cada longitud de onda presenta una capacidad de penetración diferente, que se debe considerar para no presentar efectos colaterales indeseables sobre los tejidos. Por ejemplo, una longitud de onda afín a los cromóforos que penetre sobre hueso puede producir necrosis $[1,2,5,10]$.

\section{Longitud de extinción}

Adicionalmente, Coluzzi y Convissar [1,2,5,10] proponen el concepto de longitud de extinción a la propiedad que combina la absorción y la penetración del láser en el tejido. Esta propiedad fundamenta que el grosor de una sustancia absorbe el 98 \% de la energía del láser. Así, a mayor longitud de extinción, menor absorción y mayor penetración. Por el contrario, a menor longitud de extinción, mayor absorción y menor penetración. Los láseres afines al agua como el Er:YAG y el de dióxido de carbono son afines al agua, por lo que presentan una longitud de extinción baja, porque presentan baja penetración en tejidos blandos. Otros láseres de menor longitud de onda, como el de neodimio, tienen alta penetración por su afinidad a los pigmentos [5].

\section{CONCLUSIONES}

Una variedad de longitudes de onda de láser se utiliza en odontología. El presente trabajo es un punto inicial de referencia acerca de los diferentes parámetros del láser que se deben considerar. Ellos incluyen la longitud de onda del láser y su afinidad respectiva, además de cómo esta luz amplificada actúa sobre los tejidos a partir de ciertas características (absorción, penetración, etc.), para producir efectos diferentes.

No todos los láseres actúan igual y una misma longitud de onda puede tener interacciones diferenciales en los tejidos si se utilizan estos parámetros diferencialmente. Por ello, este documento busca ser una apertura al nuevo paradigma de la odontología láser como modalidad terapéutica. Existen varios documentos que evidencian la efectividad del uso del láser en varias especialidades de la odontología y sus posibles líneas de investigación [32].

\section{RECOMENDACIONES}

Este documento sobre las bases biológicas y físicas del láser en odontología es un punto de partida para la discusión académica y clínica pertinente sobre los alcances de la odontología láser como modalidad terapéutica. Se recomienda identificar documentos que aporten evidencia sólida para orientar los alcances clínicos reales de los efectos clínicos a corto y largo plazo.

\section{REFERENCIAS}

1. Coluzzi D. Fundamentals of dental lasers: science and instruments. Dent Clin North Am. 2004; 48: 751-70.

2. Coluzzi D. Fundamentals of lasers in dentistry: basic science, tissue interaction, and instrumentation. J Laser Dent. 2008; 16(S): 4-10.

3. Myers TD, Sulewski GJ. Evaluating dental lasers: what the clinician should know. Dent Clin North Am. 2004; 48: $1127-44$. 
4. Maggioni M, Attanasio T, Scarpelli F. Láser en odontología. Caracas, Venezuela: Amolca; 2001.

5. Convissar R. Principles and practice of laser dentistry. New York, NY: Mosby Elsevier; 2011.

6. Scala J. El láser: instrumento clave en la ciencia y tecnología modernas. En: Ocaña JL, Yáñez AJ, editores. El láser y sus aplicaciones en el ámbito industrial. La Coruña, España: Servicio de Publicaciones Universidade da Coruña; 2000. pp. 13-62.

7. Dederich D, Bshick R. Lasers in dentistry: separating science from hype. J Am Dent Assoc. 2004; 135: 204-12.

8. Londoño O, Maldonado L, Calderón L. Guía para construir estados del arte. Bogotá, Colombia: International Corporation of Networks of Knowledge; 2014.

9. Rioja C, Allepuz C, Rioja L. Láser. Clin Urol Complut.1994; 3: 499-508.

10. Convissar R. The biologic rationale for the use of lasers in dentistry. Dent Clin North Am. 2004; 48: 771-94.

11. Smith K. The photobiological basis of low level laser therapy. Laser Ther. 1991; 3: 19-24.

12. Weiner G. Laser dentistry practice management. Dent Clin North Am. 2004; 48: 1105-26.

13. Zandparsa R. Latest biomaterials and technology in dentistry. Dent Clin North Am. 2014; 58: 113-34.

14. Ishikawa I, Aoki A, Takasaki A, Mizutani K, Sasaki K, Izumi Y. Application of lasers in periodontics: True innovation or myth? Periodontology 2000. 2009; 50: 90-126.

15. Aoki A, Sakaki K, Watanabe H, Ishikawa I. Lasers in nonsurgical periodontal therapy. Periodontology 2000. 2004; 36: 59-97.

16. Pang P, Andreana S, Aoki S, Coluzzi D, Obeidi A, Olivi G, Parker S, Rechmann P, Sulwski J, Sweeney C, Swick M, Yung F. Laser energy in oral soft tissue applications: position paper. J Laser Dent. 2010; 18(3): 123-31.

17. Trullols C, España A, Aytés L, Gay C. Aplicaciones del láser blando en odontología. Anales Odontoestomatol. 1997; 2: 45-51.

18. Jacques S. Optical properties of biological tissues: a review. Phys Med Biol. 2013; 58: R37-61.

19. Green J, Weiss A, Stern A. Lasers and radiofrequency devices in dentistry. Dent Clin North Am. 2011; 55: 585-97.

20. Jung GI, Kim JS, Lee TH, Choi JH, Oh HB, Kim AH, Kim JS, Park JR, Chung SC, Yeom DI, Kim HS, Jun JH. Photomechanical effect on type I collagen using pulsed diode laser. Technol Health Care. 2015; 23: S535-41.

21. Moritz A, Schoop U, Goharkhay K, Schauer P, Doertbudak O, Wernisch J, Sperr W. Treatment of periodontal pockets with a diode laser. Lasers Surg Med. 1999; 22: 302-11.

22. Gregg II RH. The LANAP Protocol: laser-assisted new attachment procedure. Dentaltown. 2012 Feb: S26-8.

23. Cobb C, Low S, Coluzzi D. Lasers and the treatment of chronic periodontitis. Dent Clin North Am. 2010; 54: 35-53.

24. de Paula Eduardo C, Aranha AC, Simões A, Bello-Silva MS, Ramalho KM, Esteves-Oliveira M, de Freitas PM, Marotti J, Tunér J. Laser treatment of recurrent herpes labialis: a literature review. Lasers Med Sci. 2014; 29(4): 1517-29.

25. Flax H. Soft and hard tissue management using lasers in esthetic restoration. Dent Clin North Am. 2011; 55: 383-402.

26. Mârțu S, Amălinei C, Tatarciuc M, Rotaru M, Potârnichie O, Liliac L, Căruntu ID. Healing process and laser therapy in the superficial periodontium: a histological study. Rom J Morphol Embryol. 2012; 53(1): 111-6.

27. Romanos G, Nentwig G. Diode laser $(980 \mathrm{~nm})$ in oral and maxillofacial surgical procedures: Clinical observations based on clinical applications. J Clin Laser Med Surg. 1999; 17(5): 193-7.

28. Shankar B, Neetha R, Kumar S, Saritha G, Reddy J. Chronic inflammatory gingival overgrowths: laser gingivectomy and gingivoplasty. J Int Oral Health. 2013; 5(1): 83-7.

29. Sobouti F, Rakhshan V, Chiniforush N, Khatami M. Effects of laser-assisted cosmetic smile lift gingivectomy on postoperative bleeding and pain in fixed orthodontic patients: a controlled clinical trial. Prog Orthod. 2014; 15: 66. 
30. Martins MR, Carvalho MF, Vaz IP, Capelas JA, Martins MA, Gutknecht N. Efficacy of Er,Cr:YSGG laser with endodontical radial firing tips on the outcome of endodontic treatment: blind randomized controlled clinical trial with six-month evaluation. Lasers Med Sci. 2013; 28(4): 1049-55.

31. Adams T, Pang P. Lasers in aesthetic dentistry. Dent Clin North Am. 2004; 48: 833-60.

32. Rechmann P. Dental laser research: selective ablation of caries, calculus, and microbial plaque from the idea to the first in vivo investigation. Dent Clin North Am. 2004; 48: 1077-104.

\section{Notas}

* Artículo de investigación

Licencia Creative Commons CC BY 4.0

Cómo citar este artículo: Briceño JF, Gaviria DA, Carranza YA. Láser en odontología: fundamentos físicos y biológicos. Univ Odontol. 2016 Jul-Dic; 35(75): 1-10. https://www.doi.org/10.11144/ Javeriana.uo35-75.loff 